\title{
Cinétique d'accumulation et rétention d'espèces du mercure chez l'étoile de mer Leptasterias polaris: une expérience de transfert trophique à long terme Uptake kinetics and retention of mercury species in starfish Leptasterias polaris: a long term trophic transfer experiment
}

\author{
E. Pelletier et S. Maheu
}

Volume 9, numéro 3, 1996

URI : https://id.erudit.org/iderudit/705257ar

DOI : https://doi.org/10.7202/705257ar

\section{Aller au sommaire du numéro}

\section{Éditeur(s)}

Université du Québec - INRS-Eau, Terre et Environnement (INRS-ETE)

\section{ISSN}

0992-7158 (imprimé)

1718-8598 (numérique)

\section{Découvrir la revue}

\section{Citer cet article}

Pelletier, E. \& Maheu, S. (1996). Cinétique d'accumulation et rétention d'espèces du mercure chez l'étoile de mer Leptasterias polaris: une expérience de transfert trophique à long terme. Revue des sciences de l'eau / Journal of Water Science, 9(3), 351-364. https://doi.org/10.7202/705257ar

\section{Résumé de l'article}

Dans le cadre de travaux sur le rôle des échinodermes dans le cycle biogéochimique de certains métaux traces en milieu côtier, une étude de l'accumulation et de la rétention d'espèces chimiques du mercure a été menée avec l'étoile de mer Leptasterias polaris sur une période de 40 jours. Les étoiles de mer ont été divisées en deux groupes et nourries avec des moules contaminées soit au méthylmercure (MeHg) (14 mg.kg-1), soit au mercure inorganique (13 mg.kg-1). Les résultats montrent une vitesse d'accumulation dans le système digestif de $0,22 \mathrm{mg} . \mathrm{kg}-1 . \mathrm{j}-1$ pour le $\mathrm{Hg}$ inorganique et 0,17 mg.kg-1.j-1 pour le MeHg. Ces vitesses sont 10 à 15 fois moins importantes dans les gonades et l'endosquelette. Pour le Hg inorganique, la charge relative dans les différents organes analysés se fixe après quelques jours et demeure stable jusqu'à la fin de l'expérience. Pour le MeHg, au contraire, cette charge relative passe progressivement du système digestif vers les gonades et l'exosquelette tout au long de l'expérience. Le pourcentage de rétention (\%) demeure constant pour le $\mathrm{Hg}$ inorganique $(51 \pm 13 \%)$ mais il augmente régulièrement pour le $\mathrm{MeHg}$, atteignant $90-95 \%$ vers la fin de l'exposition. L'application d'un modèle cinétique simple, basé sur un processus d'échange ionique, a permis de calculer des constantes de vitesse d'échange entre le digestat et le tissu digestif. Le MeHg s'échange plus vite entre la solution et les sites, mais semble mettre un peu plus de temps à voyager jusqu'aux organes de bioaccumulation. En conclusion, l'étoile L. polaris, par sa taille, sa longévité et sa grande efficacité à digérer tout ce qui est ingéré, semble en mesure de jouer un rôle important à la fois dans la séquestration du MeHg et la remise en solution du Hg inorganique. 


\title{
Cinétique d'accumulation et rétention d'espèces du mercure chez l'étoile de mer Leptasterias polaris : une expérience de transfert trophique à long terme
}

\author{
Uptake kinetics and retention of mercury species \\ in starfish Leptasterias polaris: \\ a long term trophic transfer experiment
}

\section{E. PELLETIER ${ }^{1 *}$, S. MAHEU ${ }^{2}$}

Reçu le 31 octobre 1995, accepté le 22 mars 1996.

In spite of a large body of work on the uptake of trace metals in aquatic organisms in the last two decades, very little attention has been devoted to echinoderms (urchins, starfish, and sea cucumbers, among the most important ones), a large group of invertebrates forming a major component of most coastal ecosystems. In the course of our research program on the role of echinoderms in the biogeochemical cycling of some trace metals in coastal environment, an experiment has been conducted on the uptake and the retention of mercury species with starfish Leptasterias polaris over a 40-day exposure period.

Starfish were caught near Pointe-au-Père (Québec, Canada) and were acclimatized to Jaboratory conditions for 10 days in a flow-through aquarium. Forty stars (84 $\pm 14 \mathrm{~g}$ ) were divided in two groups, kept in two aquariums, one for inorganic mercury and the other for methylmencury exposure. Animals were isolated from each other by mean of small $65-\mathrm{mm}$ net cubicles allowing free circulation of seawater at a rate of $2 \mathrm{~L} \cdot \mathrm{min}^{-1}$. This flow rate provided a rapid renewal of seawater and ensured the elimination of any soluble mercury excreted during the course of the experiment. Control starfish receiving only uncontaminated mussels were also maintained in both aquariums to monitor the possible uptake of mercury from seawater. Blue mussels were collected in the vicinity of Pointe-au-Père and were contaminated following a technique described elsewhere (PELLETIER and LAROCQUE, 1987). Average levels of inorganic mercury and methylmercury (MeHg) in mussels were $13 \pm 3$ and $14 \pm 4 \mathrm{mg} \cdot \mathrm{kg}^{-1}$, respectively. Each starfish received one contaminated mussel per day over a 40-day period. Mussejs were weighed before to be introduced in cages and their empty shells weighed again after ingestion by starfish in order to evaluate the amount of food and mercury taken up daily by each star. Two starfish were sampled in each aquarium every five days and dissected for pylo-

1. Institut national de la recherche scientifique, INRS-Océanologie.

2. Université đu Québec à Rimouski, département d'océanographie, Centre Océanologique de Rimouski, 310 Allée des Ursulines, Rimouski, (0c), Canada, G5L 3A1.

* Auteur à qui adresser ta correspondance.

* * Les commentaires seront reçus jusqu'au 15 juin 1997. 
ric caeca, gonads, and endoskeleton. Coelomic fluid was also collected. Both mercury species were analysed as total mercury (assuming a preservation level of $\pm 85-90 \%$ of the speciation during the course of the experiment) in biological tissues by cold vapour atomic adsorption spectrophotometry (detection limit $\left.=0.005 \mathrm{mg} \cdot \mathrm{kg}^{-1} \mathbf{w w}\right)$. The coefficient of variation was $\pm 15 \%$ and the recovery yield of MeHg-spiked samples was $92 \pm 12 \%$.

The concentration of inorganic mercury reached $7.56 \mathrm{mg} \cdot \mathrm{kg}^{-1}$ (wet weight) in pyloric caeca and the uptake rate was $0.22 \mathrm{mg} \cdot \mathrm{kg}^{-1} \cdot \mathrm{d}^{-1}$. Concentrations in gonads and endoskeleton were 10 to 20 times lower than in caeca (Fig. I). The uptake rate of $\mathrm{MeHg}\left(0.17 \mathrm{mg} \cdot \mathrm{kg}^{-1} \mathrm{~d}^{-1}\right)$ was slightly slower and the maximum concentration reached in caeca was $5.34 \mathrm{mg} \cdot \mathrm{kg}^{-1}$. Mercury concentrations found in coelomic fluid were low and at least 100 times smaller than those in ceaca. No mercury was found in tissues of control starfish indicating that mercury excreted by diffusion in water by contaminated starfish was not re-adsorbed by other starfish in aquariums. The mercury load in each organ of stars was calculated and expressed as a pencent (\%) of total $\mathrm{Hg}$ uptake for each chemical species (Fig. 2). The inorganic $\mathrm{Hg}$ loads in pyloric caeca, gonads and endoskeleton reached a steady-state after only 10 days and remained unchanged up to the end of the experiment. The behaviour of MeHg was totally different as the load in caeca decreased from $95 \%$ to $65 \%$ but increased from almost zero up to $30 \%$ in endoskeleton. Finally, the retention (\%) of mercury species was calculated by dividing the actual total amount of mercury in each starfish by the total amount of mercury received from mussels (Fig. 3). The retention of inorganic $\mathrm{Hg}$ was about $50 \%$ throughout the exposure period whereas the retention of $\mathrm{MeHg}$ increased up to $90-95 \%$ at the end of the experiment.

A kinetic model, based upon the assumption that the uptake process of $\mathrm{Hg}$ species in the digestive system is quite similar to an ion-exchange adsorption mechanism between a contamination solution and a solid surface, was developed. The integrated equation of the rate law was expressed as :

$$
\frac{\ln \left(C_{0} m-q\right)}{q_{0}-q}=k^{+}\left(C_{0} m-q_{0}\right) t+\ln \left(\frac{C_{0} m}{q_{0}}\right)
$$

were $C_{0}$ is the initial concentration of mercury in the prey, $q_{0}$ is the maximun amount of mercury being absorbed, and $m$ stands for the wet weight of the animal. This equation allowed the plot of its left-hand side against time and the slope provided an estimation of an apparent exchange rate constant for each mercury species (Fig. 4). The rate constant $\mathrm{k}^{\prime} \mathrm{MeHg}$ was slightly higher than $\mathrm{k}^{\prime} \mathrm{Hg}$, indicating a faster exchange rate for $\mathrm{MeHg}$ between digested mussel tissues and binding sites (and also between sites) in caeca. However, a faster exchange rate do not mean a faster uptake rate because transport rate towards other organs also play an important role in the whole bioaccumulation process.

In conclusion, starfish, by its ability to digest all ingested tissues, seems to be in a position to play a major role in the sequestration of methylmercury (high retention) from mussels and from other potential preys and in recycling inorganic mercury in solution (low retention). Starfish can be seen as « marine digester * which engulfs bivalves and other invertebrates (following species) and sends back soluble metabolites and trace metals which have not been bioaccumulated.

Key words: echinoderms, starfish mercury, methylmercury, uptake, retention, ionexchange rate, kinetic model, constant rate.

Dans le cadre de travaux sur le rôle des échinodermes dans le cycle biogéochimique de certains métaux traces en milieu côtier, une étude de l'accumulation 
et de la rétention d'espèces chimiques du mercure a été menée avec l'étoile de mer Leptasterias polaris sur une période de $\mathbf{4 0}$ jours. Les étoiles de mer ont été divisées en deux groupes et nourries avec des moules contaminées soit au mêthylmencure $(\mathrm{MeHg})\left(14 \mathrm{mg} \cdot \mathrm{kg}^{-1}\right)$, soit au mercure inorganique $\left(13 \mathrm{mg} \cdot \mathrm{kg}^{-1}\right)$. Les résultats montrent une vitesse d'accumulation dans le système digestif de $0,22 \mathrm{mg} \cdot \mathrm{kg}^{-1} \cdot \mathrm{j}^{-1}$ pour le $\mathrm{Hg}$ inorganique et $0,17 \mathrm{mg} \cdot \mathrm{kg}^{-1} \cdot \mathrm{j}^{-1}$ pour le MeHg. Ces vitesses sont 10 à 15 fois moins importantes dans les gonades et l'endosquelette. Pour le $\mathrm{Hg}$ inorganique, la charge relative dans les différents organes analysés se fixe après quelques jours et demeure stable jusqu'à la fin de l'expérience. Pour le $\mathrm{MeHg}$, au contraire, cette charge relative passe progressivement du système digestif vers les gonades et l'exosquelette tout au long de l'expérience. Le pourcentage de rétention (\%) demeure constant pour le $\mathbf{H g}$ inorganique $(51 \pm 13 \%)$ mais il augmente régulièrement pour le MeHg, atteignant $90-95 \%$ vers la fin de l'exposition. L'application d'un modèle cinétique simple, basé sur un processus d'échange ionique, a permis de calculer des constantes de vitesse d'échange entre le digestat et le tissu digestif. Le MeHg s'échange plus vite entre la solution et Jes sites, maís semble mettre un peu plus de temps à voyager jusqu'aux organes de bioaccumulation. En conclusion, l'étoile $L$. polaris, par sa tailie, sa longévité et sa grande efficacité à digérer tout ce qui est ingéré, semble en mesure de jouer un rôle important à la fois dans la séquestration du $\mathrm{MeHg}$ et la remise en solution du $\mathrm{Hg}$ inorganique.

Mots-clés: échinodermes, étoile de mer, mercure, méthylmercure, prise en charge. rétention, échange ionique, modèle cinétique, constante de visesse.

\section{INTRODUCTION}

La bioaccumulation du mercure inorganique et du méthylmercure $(\mathrm{MeHg})$ en milieu aquatique a été abondamment étudiée au cours des dernières années. Chez les invertébrés marins, les travaux expérimentaux et de terrain ont surtout porté sur les filtreurs comme la moule Mytilus edulis (PELLETIER, 1986 ; RIISGARD et HANSEN, 1990), la mye Macoma balthica (RIISGAARD et al., 1985) et l'huître Crassostrea virginica (PALMER et al., 1993) et sur les crustacés, tels les crevettes Pandalus borealis (ROULEAU et al., 1992) et Crangon crangon (RIISGAARD et FAMME, 1986) et l'écrevisse Orconectes propinquus (WRIGHT et al., 1991). Par contre, la bioaccumulation du mercure et des autres métaux traces chez les échinodermes (oursins, étoiles et holothuries, parmi les plus importants), une composante essentielle des écosystèmes côtiers, demeure mal connue et le rôle de ces espèces est encore difficile à cerner quant à leur contribution aux cycles biogéochimiques de ces métaux. On ne trouve que peu d'études portant sur la bioaccumulation des métaux (GUARY et al., 1982 ; BJERREGARD, 1988 ; DEN BESTEN et al., 1989 ; WARNAU et al., 1995a, 1995b) et du mercure en particulier (IGLESIAS et PENCHASZADECH, 1983, SORRENSEN et BJERRAGARD, 1991) chez les échinides. Notre laboratoire s'est intéressé depuis plusieurs années à l'étoile de mer, Leptasterias polaris, une espèce répandue le long des côtes de l'Atlantique nord s'adaptant facilement aux conditions de laboratoire et jouant le rôle de prédateur supérieur dans la chaîne trophique côtière : particules $\rightarrow$ moules $\rightarrow$ étoiles de mer. Une première expérience, menée par PELLETIER et LAROCQUE (1987), a montré que les étoiles de mer accumulaient rapidement le méthylmercure via 
cette chaîne alimentaire. Des études subséquentes ont porté sur les processus à très court terme (24-48 h) de distribution et d'excrétion du mercure inorganique et du méthylmercure (MAHEU et PELLETIER, 1995) ainsi que sur la cinétique de bioaccumulation et du transfert entre les compartiments de l'animal (ROULEAU et al., 1995).

La présente expérience avait pour but de poursuivre ces travaux sur $L$. polaris en comparant la prise en charge et la rétention dans différents organes de l'animal du mercure inorganique et du méthylmercure via l'alimentation sur une période de 40 jours. Le mécanisme de bioaccumulation des deux espèces mercuriques est discuté à la fumière d'une approche cinétique simple faisant appel à un processus d'échange toniquue dans le système digestif.

\section{MATÉRIEL ET MÉTHODES}

Les étoiles de mer utilisées pour cette expérience ont été prélevées en plongée sur la côte sud de l'estuaire du Saint-Laurent à la hauteur de Pointe-au-Père (Québec). Parmi les animaux prélevés, un groupe de 40 étoiles ayant une bonne uniformité de poids ( $84 \pm 18 \mathrm{~g})$ a été distribué dans deux aquariums $(530 \mathrm{~L})$ munis de casiers individuels $(20 \times 26 \mathrm{~cm})$ en filet de maille $65 \mathrm{~mm}$ permettant d'isoler les étoiles les unes des autres tout en les gardant dans des conditions environnementales identiques. Chaque aquarium était équipé de deux aérateurs et d'une circulation constante en eau de mer (28-30 psu) de $2 \mathrm{~L} \cdot \mathrm{min}^{-1}$ maintenue pendant la durée de l'expérience pour s'assurer que toute excrétion de mercure par les étoiles soit éliminée par le renouvellement rapide de l'eau et ainsi d'éviter toute possibilité de réadsorption externe du mercure.

\section{PROCÉDURE DE CONTAMINATION}

Des moules, Mytilus edulis, de $35 \pm 3 \mathrm{~mm}$ ont été prélevées en zone intertidale non contaminée par le mercure à quelques $\mathrm{km}$ du lieu de prélèvement des étoiles et ont été placées dans des bacs de contamination (un pour chaque espèce de mercure) avec un débit d'eau de $200 \mathrm{~mL} \cdot \mathrm{min}^{-1}$ et la contamination a été effectuée selon le protocole déjà décrit par PELLETIER (1986). Les concentrations de mercure y étaient de $30 \mu \mathrm{g} \cdot \mathrm{L}^{-1}(150 \mathrm{nM})$ pour $\mathrm{HgCl}_{2}$ et de $6 \mu \mathrm{g} \cdot \mathrm{L}^{-1}$ (28 nM) pour $\mathrm{CH}_{3} \mathrm{HgOH}$. Après 5 jours de contamination, les niveaux atteignaient $13 \pm 3 \mathrm{mg} \cdot \mathrm{kg}^{-1}(\mathrm{n}=10)$ pour les moules exposées au $\mathrm{Hg}$ inorganique et $14 \pm 4 \mathrm{mg} \cdot \mathrm{kg}^{-1}(\mathrm{n}=10)$ pour les moules exposées au méthylmercure. Les moules contaminées étaient conservées dans des bacs à circulation continue d'eau de mer non contaminée et les stocks étaient renouvelés tous les 5 jours. Aucun changement significatif des concentrations en mercure dans les moules et de la spéciation n'a été observé pendant cette période confirmant les travaux de ROULEAU et al. (1992) qui ont montré que les moules ne dé-méthylent pas signifi- 
cativement le méthylmercure sur une période d'au moins 13 jours. D'autre part, les travaux de RIISGARD et al. (1985) ont aussi montré que la dépuration des espèces du mercure chez $M$. edulis était très lente, nécessitant plusieurs semaines avant qu'une différence significative ne soit observée.

Après une période de jeûne de 10 jours, les étoiles de mer ont été nourries avec les moules contaminées, à raison d'une moule par étoile et par jour. Les moules étaient préalablement pesées et les coquilles vides étaient récupérées et pesées afin de déterminer la masse de chair ingérée par chaque étoile ainsi que la charge reçue en mercure. De la même façon, quatre étoiles témoins (deux dans chacun des aquariums) étaient nourries avec des moules non contaminées. De façon générale, les étoiles s'alim̄entaient régulièrement chaque jour mais il arrivait sporadiquement que certains étoiles ne s'alimentent pas. La moule donnée la veille était alors retirée de la cage et une nouvelle moule étaít offerte. Aucun décès n'a été observé pendant la durée de l'expérience. La température de l'eau a augmenté lentement de $8^{\circ} \mathrm{C}$ à $13,5^{\circ} \mathrm{C}$ pendant les 40 jours de bioaccumulation.

Tous les cinq jours, deux étoiles par aquarium ont été échantillonnées au hazard et disséquées de façon à prélever l'ensemble des caeca pyloriques et des gonades ainsi que des échantillons de l'endosquelette et du liquide coelomique, ce dernier étant prélevé à l'aide d'une seringue hypodermique insérée dans l'un des bras de l'animal avant la dissection. Les échantillons ont été conservés à $-20^{\circ} \mathrm{C}$ jusqu'à l'analyse du mercure dans les organes et tissus. Les témoins ont été prélevés au jour 40 seulement.

\section{Analyse du mercure}

De multiples travaux de laboratoire ont montré que seules les bactéries sont en mesure de méthyler ou dé-méthyler le mercure (PELLETIER, 1995). A de nombreuses reprises nous avons tenté d'observer la méthylation du mercure chez les moules (ROULEAU et al., 1992), les crevettes et les étoiles de mer, sans succès. Comme les échinodermes ne semblent disposer d'aucune capacité particulière de méthyler ou de dé-méthyler le mercure, les concentrations en $\mathrm{Hg}$ total observées dans les divers tissus analysés ont été attribuées aux espèces utilisées lors de la contamination. Ceci n'exclut pas la possibilité d'un petit pourcentage de méthylation du mercure dans le système digestif des moules et des étoiles provoqué par les bactéries qui s'y trouveraient mais ce pourcentage (peut-être 10-15\% après plusieurs semaines selon nos observations chez la moule) est de toute façon égal ou inférieur à la variabilité analytique observée. Sur cette base, il a été décidé d'analyser le mercure total seulement et de poser l'hypothèse que la quantité totale de mercure mesurée correspondait essentiellement à l'espèce de mercure utilisée pour la contamination.

Le mercure total a donc été analysé en duplicata dans les moules et les organes de l'étoile de mer selon la méthode décrite par HATCH et OTT (1968). Les tissus ont d'abord subi une digestion à $45^{\circ} \mathrm{C}$ dans l'acide nitrique concentré pendant $12 \mathrm{~h}$ dans des ballons de verre fermés et le mercure total a été analysé par absorption atomique en vapeur froide (Perkin-Elmer HG-3). Le coefficient de variation de cette méthode était de $\pm 15 \%$ et sa limite de détection de $0,005 \mathrm{mg} \cdot \mathrm{kg}^{-1}$ en poids humide. Le taux de récupération des ajouts internes de méthylmercure à des échantillons de tissu biologique était de $92 \pm 12 \%$. 


\section{RÉSULTATS}

\section{Accumulation de $\mathrm{Hg}$ inorganique}

Les données de bioaccumulation du mercure inorganique dans les organes et tissus de $L$. polaris pendant les 40 jours de contamination se retrouvent à la figure 1 . Les valeurs sont rapportées pour les caeca pyloriques (appareil digestif de l'étoile situé dans chacun de ses bras et relié au disque central et à son estomac), les gonades (appareil reproducteur aussi localisé dans chacun des bras et relié au disque central) et l'endosquelette (enveloppe formé de tissus conjonctifs et de plaquettes calcaires entourant complètement l'animal). La bioaccumulation du $\mathrm{Hg}$ inorganique s'est faite selon le même profil dans tous les organes analysés. La bioaccumulation a été pratiquement nulle pour les dix premiers jours de l'exposition mais on observe ensuite une période d'accumulation rapide et linéaire s'étendant sur le reste de l'expérience. Les concentrations en mercure n'ont pas atteint de plateau. Les concentrations les plus élevées se sont retrouvées dans les caeca pyloriques $\left(7,56 \mathrm{mg} \cdot \mathrm{kg}^{-1}\right)$ où la bioaccumulation s'est faite au rythme de $0,22 \mathrm{mg} \cdot \mathrm{kg}^{-1} \cdot \mathrm{j}^{-1}$ $\left(r^{2}=0,95\right)$. Les concentrations retrouvées dans les gonades $\left(0,8 \mathrm{mg} \cdot \mathrm{kg}^{-1}\right)$ et l'endosquelette $\left(0,42 \mathrm{mg} \cdot \mathrm{kg}^{-1}\right)$ étaient de 10 à 20 fois plus faibles que celles retrouvées dans les caeca pyloriques au même moment. La vitesse de prise en charge y était donc beaucoup plus lente avec des valeurs de $0,022 \mathrm{mg} \cdot \mathrm{kg}^{-1} \cdot \mathrm{j}^{-1}\left(\mathrm{r}^{2}=0,93\right)$ dans les gonades et $0,012 \mathrm{mg} \cdot \mathrm{kg}^{-1} \cdot \mathrm{j}^{-1}\left(\mathrm{r}^{2}=0,97\right)$ dans l'endosquelette.

II est à noter qu'aucune quantité mesurable de mercure n'a èté retrouvée dans les organes et tissus des quatre étoiles témoins indiquant f'absence de contamination provenant de l'eau des aquariums et des déjections des étoiles contaminées.

\section{Accumulation de méthylmercure}

La bioaccumulation sur 40 jours du méthylmercure dans les organes de L. polaris est présentée dans la partie droite de la figure 1. D'une façon générale, la prise en charge du $\mathrm{MeHg}$ a été moins régulière que celle du $\mathrm{Hg}$ inorganique conduisant à des corrélations plus faibles des concentrations en fonction du temps. Dans les caeca pyloriques, la bioaccumulation a débuté plus tôt que celle du $\mathrm{Hg}$ inorganique mais la vitesse moyenne $\left(0,17 \mathrm{mg} \cdot \mathrm{kg}^{-1} \cdot \mathrm{j}^{-1}, \mathrm{r}^{2}=0,85\right)$ a été un peu plus lente. Tout comme pour le $\mathrm{Hg}$ inorganique, la bioaccumulation dans les gonades et l'endosquelette a débuté après 10 jours avec des vitesses respectives de $0,027 \mathrm{mg} \cdot \mathrm{kg}^{-1} \cdot \mathrm{j}^{-1}\left(\mathrm{r}^{2}=0,90\right)$ et de $0,017 \mathrm{mg} \cdot \mathrm{kg}^{-1} \cdot \mathrm{j}^{-1}\left(\mathrm{r}^{2}=0,65\right)$, un peu plus élevées que celles observées pour le $\mathrm{Hg}$ inorganique. De nouveau, les concentrations retrouvées dans ces deux organes sont environ dix fois moins élevées que dans les caeca pyloriques.

\section{Le liquide coelomique}

Les concentrations de mercure inorganique ou de méthylmercure dans le liquide coelomique sont demeurées très faibles pendant toute l'expérience et ont été plus de 100 fois plus faibles que celles retrouvées dans les caeca, atteignant à peine une valeur de $0,023 \mathrm{mg} \cdot \mathrm{kg}^{-1}$ pour le $\mathrm{Hg}$ inorganique et de $0,017 \mathrm{mg} \cdot \mathrm{kg}^{-1}$ pour l'exposition au MeHg. Aucune corrélation significative n'a été observée entre les concentrations et le temps écoulé. 

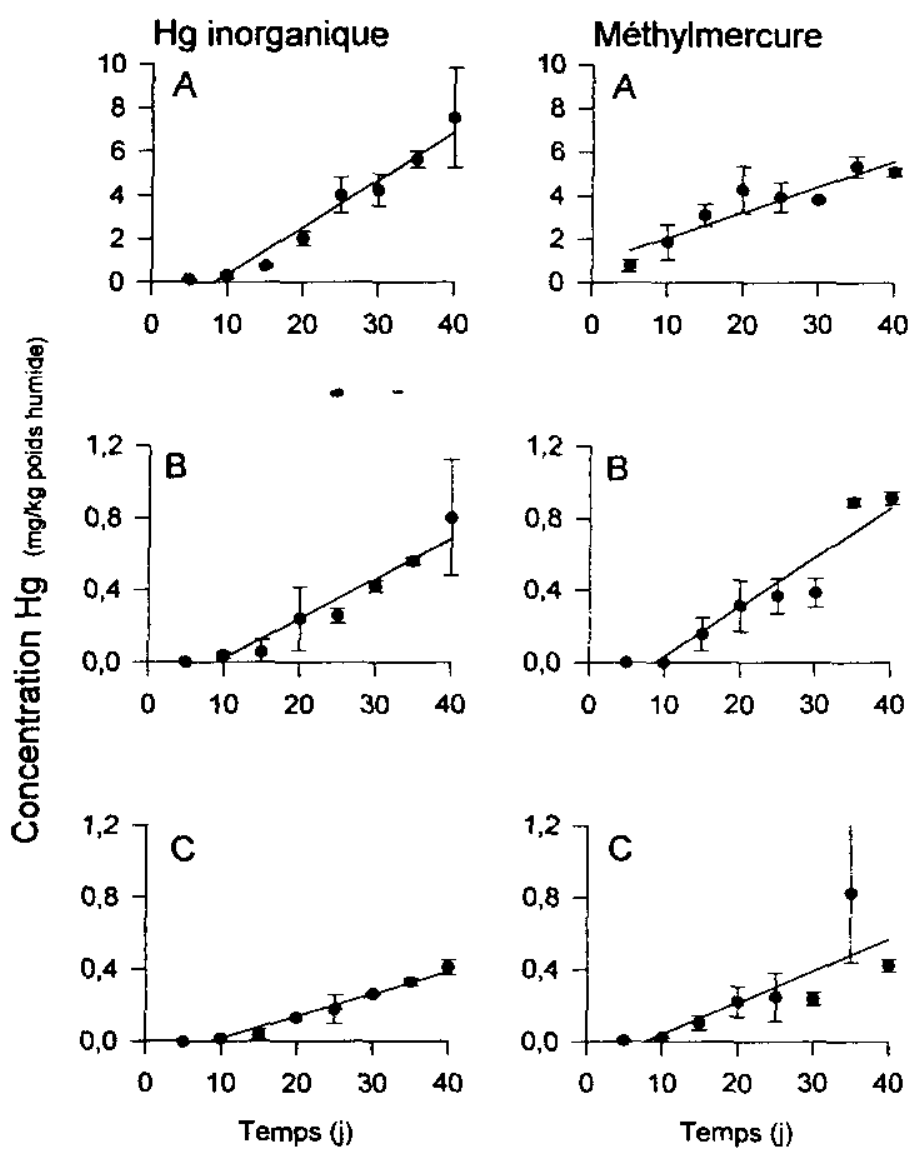

Figure 1

Concentrations des espèces de mercure (analysées sous forme de mercure total en $\mathrm{mg} \cdot \mathrm{kg}^{-1}$ poids humide) dans (A) les caeca pyloriques, (B) les gonades et $(C)$ l'endosquelette de l'étoile de mer $L$. polaris nourrie des moules contenant $13 \pm 3 \mathrm{mg} \cdot \mathrm{kg}^{-1}$ de $\mathrm{Hg}$ inorganique ou $14 \pm 4 \mathrm{mg} \cdot \mathrm{kg}^{-1} \mathrm{de}$ méthylmercure selon le cas sur une période de 40 jours.

Concentrations of mercury species (analysed as total mercury in $\mathrm{mg} \mathrm{kg}^{-1}$ wet weight) in (A) pyloric caeca, $(B)$ gonads and $(C)$ endoskeleton of starfish L. polaris fed with mussels containing $13 \pm 3 \mathrm{mg} \cdot \mathrm{kg}^{-1}$ of inorganic $\mathrm{Hg}$ or 14 $\pm 4 \mathrm{mg} \cdot \mathrm{kg}^{-1}$ of methylmercury following the cas over a 40 -day period.

\section{Charge relative et rétention des espèces du mercure dans les organes}

Nous avons calculé les quantités totales de mercure retrouvées dans les organes en multipliant la concentration $\left(\mathrm{mg} \cdot \mathrm{kg}^{-1}\right)$ dans un organe donné par le poids $(\mathrm{kg})$ de cet organe, ce dernier étant calculé en mútipliant le poids de l'animal par le pourcentage moyen en poids de cet organe $(10,1 \%$ pour les caeca pyloriques, $4,3 \%$ pour les gonades et $52,2 \%$ pour l'endosquelette). Les autres organes (l'estomac $0,8 \%$ et les podia $18,0 \%$ ) et le liquide coelomique n'ont pas été utilisés pour ces calculs à cause de leur trop faible contribution $(<5 \%)$ au 
bilan total du mercure. La charge en mercure a été exprimée en pourcentage (\%) du mercure analysé dans un organe donné par rapport au total accumulé dans tout l'animal.

L'évolution de ces charges relatives en mercure inorganique et en méthylmercure dans les principaux organes de $L$. polaris est présentée à la figure 2 . La charge en $\mathrm{Hg}$ inorganique dans les caeca pyloriques est passée rapidement de $95 \%$ du total retrouvé dans l'animal au jour 5 à $76 \%$ dès le jour 10 (fig. $2 \mathrm{~A}$ ) et est ensuite demeurée alors stable pour le reste de l'expérience, à $76 \pm 3 \%$ en moyenne. Au contraire, la charge relative en méthylmercure des caeca pyloriques a décru de façon linéaire pendant toute la durée de l'expérience à une vitesse de $0,95 \% \cdot j^{-1}\left(r^{2}=0,95\right)$.
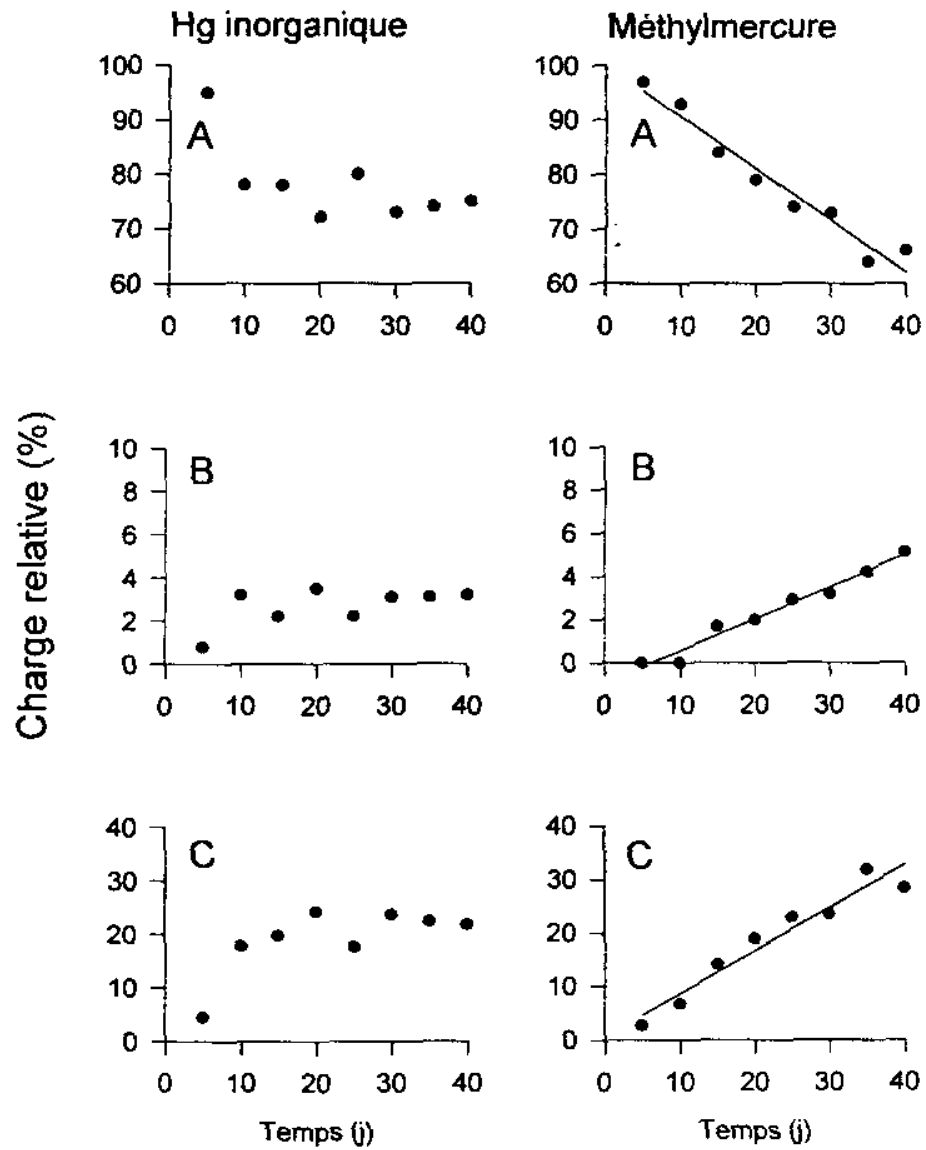

Figure 2 Variation de la charge relative en $\mathrm{Hg}$ inorganique et en méthylmercure dans (A) les caeca pyloriques, (B) les gonades et (C) l'endosquelette de l'étoile de mer $L$. polaris sur une période de 40 jours.

Variation in the relative load in inorganic $\mathrm{Hg}$ and methylmercury in $(A)$ pyloric caeca, $(B)$ gonads and $(C)$ endoskeleton de l'étoil de mer $L$. polaris over a 40-day period. 
Les charges relatives en $\mathrm{Hg}$ inorganique dans les gonades (fig. $2 B$ ) et l'endosquelette (fig. 2C) sont demeurées stables du jour 10 jusqu'à la fin de l'expérience, à $2,9 \pm 0,5 \%$ et $21 \pm 3 \%$ respectivement. Par contre la charge en $\mathrm{MeHg}$ dans les gonades a augmenté de façon linéaire $\left(r^{2}=0,97\right)$ pendant toute la durée de l'expérience à une vitesse de $0,15 \% \cdot \mathrm{j}^{-1}$. Enfin, l'endosquelette a montré un comportement similaire à celui des gonades pour le $\mathrm{MeHg}$ avec une augmentation constante tout au long de l'expérience $\left(r^{2}=0,80\right)$, passant de 2,7 à $28,4 \%$ à mesure que diminuait la charge relative dans les caeca pyloriques.

La quantité totale de mercure ingérée a été calculée en multipliant le poids de chair des moules ingérées par le niveau de $\mathrm{Hg}$ total analysé dans ces dernières ( $\mathrm{mg} \cdot \mathrm{kg}^{-1}$ ) et le pourcentage de rétention a été calculé par rapport au mercure total retrouvé dans l'ensemble des organes (fig. 3). Malgré une forte variabilité induite par l'utilisation d'individus différents à chaque prélèvement, on distingue nettement le comportement du mercure inorganique par rapport à celui du méthylmercure. II n'existe pas de relation significative $\left(r^{2}=0,06\right)$ entre le taux de rétention du $\mathrm{Hg}$ inorganique et le temps d'exposition et on observe plutôt une assez forte fluctuation autour d'une valeur moyenne de $51 \pm 13 \%$. Par contre, le pourcentage de rétention du MeHg a augmenté à un rythme de $1,36 \% \cdot j^{-1}$ $\left(r^{2}=0,77\right)$ et a atteint à la fin de l'expérience des valeurs de $90-95 \%$ indiquant que la quasi-totalité du mercure ingéré était entreposée dans les tissus de l'étoile.
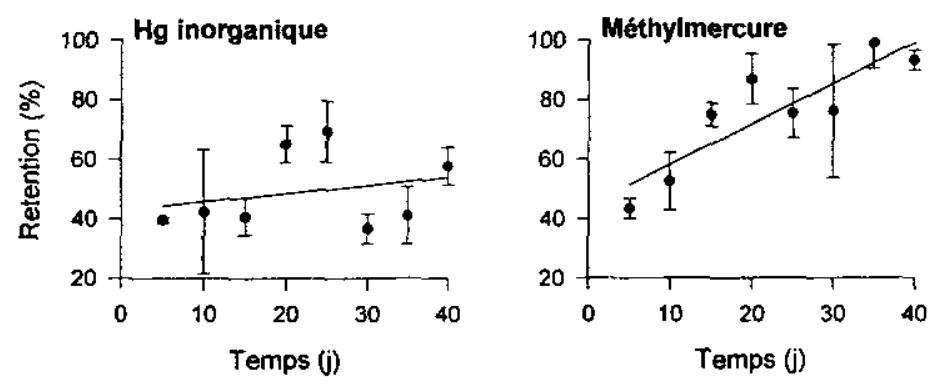

Figure 3 Evolution de la rétention (\%) du mercure inorganique et du méthylmercure par $L$. polaris sur une période de 40 jours.

Variation of the retention (\%) of inorganic $\mathrm{Hg}$ and methylmercury by L. polaris over a 40-day period.

\section{DISCUSSION}

Il y a quelques années, notre laboratoire rapportait tes premiers résultats de la bioaccumulation du méthylmercure chez $L$. polaris via son alimentation (PELLETIER et LAROCQUE, 1987). La présente expérience confirme les taux de bioaccumulation obtenus alors pour les caeca et pour l'endosquelette avec une apparente stabilisation des concentrations après trois semaines d'une alimentation continue avec les moules contaminées. L'élément nouveau ici est la constatation d'une reprise de l'accumulation au-delà des premiers 30 jours et peut-être de la présence d'un nouveau plateau à la fin de l'expérience. La différence tient proba- 
blement dans l'utilisation dans le présent cas d'une concentration en $\mathrm{MeHg}$ environ deux fois plus élevée que la première expérience, ce qui aurait forcé l'accumulation à un niveau plus élevé. Cependant, cette différence n'a pas affecté le taux de bioaccumulation dans l'endosquelette, demeuré à 0,016 $\pm 0,002 \mathrm{mg} \cdot \mathrm{kg}^{-1} \cdot \sigma^{-1}$ et fournissant une première indication que le transfert vers ce tissu n'est pas fonction uniquement de la concentration $\mathrm{MeHg}$ dans la nourriture ingérée. Comme l'expérience a été soigneusement conduite de façon à éliminer toute possibilité de contamination provenant de l'eau de mer ambiante, le $\mathrm{MeHg}$ dans l'endosquelette ne peut venir que du système biologique interne et ce système possède des vitesses limites de transport et d'entreposage qui sont fonction de la nature des barrières biologiques à traverser et de l'affinité du $\mathrm{MeHg}$ pour les tissus récepteurs (MAMEU et PELLETIER, 1995). Comparativement, la bioaccumulation du $\mathrm{Hg}$ inorganique a été plus régulière mais plus tardive, débutant seulement après le dixième jour de contamination et se poursuivant ensuite un peu plus rapidement que celle du $\mathrm{MeHg}$.

Ce temps de latence au début d'une période d'accumulation d'un métal ou d'un organométal est difficile à expliquer en terme de mécanisme de prise en charge. L'un des mécanismes de bioaccumulation d'un métal (voir TESSIER et al., 1994, pour une revue du sujet) suppose l'établissement dans le système digestif d'une compétition entre le métal associé à une particule de nature quelconque $[\mathrm{M}]_{\text {particule }}$ et les sites de prise en charge du métal dans l'organisme [S $]_{\text {organisme- }}$ Les enzymes digestives libèrent le métal qui cherche ensuite un site énergétiquement favorable [M-S]. Evidemment, ce mécanisme est fonction de la densité des sites d'adsorption mais aussi, et surtout, de la disponibilité de ces sites. On sait aussi qu'il s'établit une compétition pour les sites mais on ignore s'il faut une certaine " activation" du site pour que le mercure puisse s'y associer. Pour examiner ce problème de façon plus formelle, on peut rapprocher la prise en charge des espèces du mercure par le système digestif de l'étoile de mer du processus d'adsorption par échange ionique entre une solution contaminée et une surface solide. Dans ce modèle, il n'y a pas d'activation des sites de liaison et il s'agit simplement d'un échange basé sur l'affinité relative des ions pour [S] $]_{\text {organisme. }}$ Cette approche est particulièrement réaliste avec le système digestif de l'étoile de mer qui digère tout et n'excrète que des déchets liquides et gazeux par diffusion.

Nous proposons ici d'adapter une approche cinétique simple qui décrit ladsorption d'un cation métallique en solution sur un solide par un processus d'échange avec un autre ion déjà adsorbé, mais dont l'affinité est moindre pour le solide (MISRA et BOWEN, 1981). La présence de certains autres ions dans la solution et leur spéciation peuvent influencer les constantes de vitesses mais, pour éviter de surcharger les équations de vitesse, nous considérerons ces paramètres comme constants durant toute l'expérience.

Si C est la concentration (nmole/g) du mercure dans la source, ici la moule contaminée et digérée, et q la quantité de l'entité $\mathrm{Hg}$ (nmole) dans le tissu de l'étoile à un temps donné $t$, on peut représenter l'échange du mercure de la proie vers le caecum en fonction du temps par une équation de vitesse simple:

$$
-\frac{d C}{d t}=k^{\prime} C\left(q_{0}-q\right)
$$

où $\mathrm{q}_{0}$ est la quantité maximale pouvant être adsorbée par le caecum et $\mathrm{k}$ ' la constante de vitesse apparente. Cependant la concentration $\mathrm{C}$ à l'intérieur de la proie à un temps $t$ n'est pas disponible en cours d'expérience et il est préférable d'expri- 
mer la vitesse de bioaccumulation dans le tissu. Si on admet qu'il n'y a pas de perte au cours de la digestion (tout le $\mathrm{Hg}$ dans la proie étant rendu bio-disponible mais pas nécessairement pris en charge ou absorbé), la quantité dans la proie à un moment donné est toujours égale à $\mathrm{Cm}=\left(\mathrm{C}_{0} \mathrm{~m}-\mathrm{q}\right)$ où $\mathrm{m}$ est la masse humide $(\mathrm{g})$ de la proie et $\mathrm{C}_{0}$ la concentration initiale du mercure dans cette proie. On peut alors écrire l'équation de vitesse :

$$
\frac{d q}{d t}=k^{\prime}\left(C_{0} m-q\right)\left(\dot{q}_{0}-q\right)
$$

Si on intègre l'équation 2 , on trouve :

$$
\frac{\ln \left(C_{0} m-q\right)}{\left(q_{0}-q\right)}=k^{\prime}\left(C_{0} m-q_{0}\right) t+\ln \left(\frac{C_{0} m}{q_{0}}\right)
$$

On reconnaît ici l'expression d'une droite ayant pour pente $k^{\prime}\left(C_{0} m-q_{0}\right)$ et une ordonnée à l'origine $\ln \left(\mathrm{C}_{0} \mathrm{~m} / \mathrm{q}_{0}\right)$ correspondant aux conditions initiales $t=0$. L'intérêt principal de cette équation est qu'à partir des données expérimentales disponibles, on peut tracer le graphique de la partie gauche de l'équation en fonction de $t$ et obtenir la pente d'où on pourra tirer la constante de vitesse $k^{\prime}=$ pente $/\left(C_{0} m-q_{0}\right)$ et aussi la valeur de $C_{0} m$.

La figure 4 montre les deux droites obtenues pour les données de $\mathrm{MeHg}$ et $\mathrm{Hg}$ inorganique à notre disposition. On constate l'excellent comportement du modèle pour les données du mercure inorganique fournissant une pente de $0,164 \mathrm{j}^{-1}$ et une valeur à l'origine de 4.01 (sans unité). Le calcul de la constante de vitesse apparente $\mathrm{K}_{\mathrm{Hg}}^{\prime}$ donne $3.6 \times 10^{-4} \mathrm{nmole}^{-1} \cdot \mathrm{j}^{-1}$. Les données pour $\mathrm{MeHg}$ sont un peu plus dispersées $\left(r^{2}=0,867\right)$ mais le modèle demeure acceptable et fournit une constante de vitesse $\mathrm{k}_{\mathrm{MeHg}}^{\prime}$ de $4,9 \times 10^{-4} \mathrm{nmole}^{-1} \cdot \mathrm{j}^{-1}$. La relative dispersion des points et la présence de quelques valeurs hors échelle nous indiquent que le modèle convient moins bien au MeHg et que le processus réel d'adsorption et de transport du $\mathrm{MeHg}$ n'est pas entièrement de nature ionique.
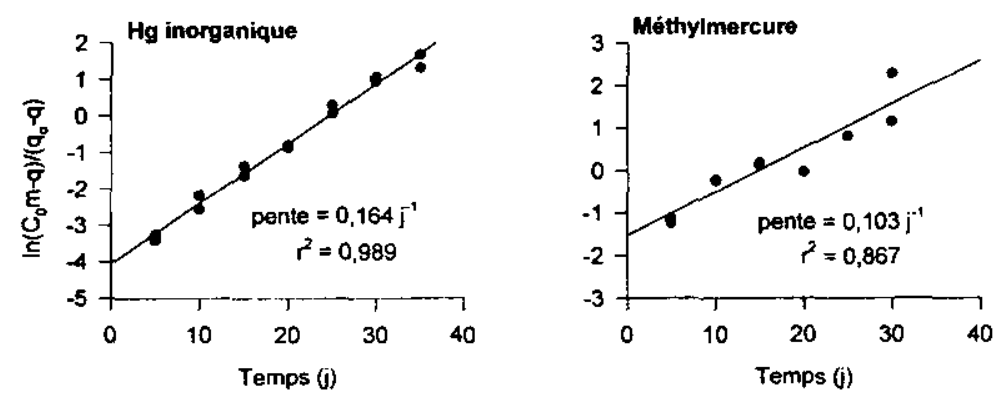

Figure 4 Graphiques de l'équation de vitesse d'échange ionique exprimée pour le $\mathrm{Hg}$ inorganique et le méthylmercure chez $L$. polaris. Toutes les données expérimentales (et non les moyennes) ont été incluses à l'exception de trois valeurs hors-échelle pour le méthylmercure.

Plots of the ion-exchange rate equation for inorganic $\mathrm{Hg}$ and methylmercury in L. polaris. All experimental data (not means) have been plotted except for three out-of-scale values for methylmercury. 
Une constante de vitesse exprime la vitesse à laquelle des échanges se produisent entre deux phases ou deux compartiments. Ainsi, une valeur de $\mathrm{k}_{\mathrm{MeHg}}$ un peu plus grande que celle de $\mathrm{k}_{\mathrm{Hg}}$ signifie que les échanges pour le $\mathrm{MeHg}$ sont plus rapides et qu'un plateau devrait être atteint plus rapidement mais ceci ne signifie pas que la bioaccumulation sera plus rapide. Un plus grand nombre d'échanges sur les sites $[S]_{\text {organisme }}$ ne signifie pas nécessairement un plus grand nombre d'ions transportés [M-S] vers l'intérieur du tissu et vers les autres organes. II est aussi intéressant de comparer ces valeurs avec celles d'un travail antérieur où nous sommes aussi parvenus à une constante de vitesse plus grande pour le transfert de $\mathrm{MeHg}$ à court terme $(48 \mathrm{~h})$ entre les compartiments de l'étoile en utilisant une approche cinétique totalement différente de celle-ci (ROULEAU et al., 1995).

A partir des valeurs à l'origine, on a pu calculer les valeurs de $C_{0} m$ à $t=0$, c'est-à-dire la quantité de mercure (nmole) que les étoiles auraient reçu au premier jour selon le modèle. Ce calcul donne une valeur d'à peine 8,2 nmoles pour le $\mathrm{Hg}$ inorganique et de $74,9 \mathrm{nmoles}$ pour le $\mathrm{MeHg}$. L'examen des données des premiers jours montre qu'effectivement les étoiles soumises à la contamination avec le $\mathrm{Hg}$ inorganique ont reçu des quantités plus faibles de mercure que celles exposées au $\mathrm{MeHg}$ parce que certaines ont refusé de manger une ou plusieurs des moules qui leur étaient données chaque jour. Par conséquent, le retard de bioaccumulation observé dans la courbe de bioaccumulation du mercure inorganique n'est pas mécanistique, mais simplement lié à un biais expérimental introduit par le comportement de certaines étoiles en début d'expérience. En bref, le modèle cinétique utilisé tend à invalider l'idée d'une activation des sites d'adsorption émise plus haut mais suggère fortement l'idée d'un mécanisme d'adsorption par échange ionique entre les sites transporteurs et le digestat contenant le métal dissous, en particulier pour le $\mathrm{Hg}$ inorganique.

La comparaison des charges relatives dans l'organe source (l'appareil digestif) et dans les organes et tissus récepteurs met en évidence toute la différence de comportement entre le $\mathrm{Hg}$ inorganique et le méthyimercure. La charge relative fournit un instantané de la distribution du métal entre les compartiments de l'animal pour chaque période d'échantillonnage. Pour le $\mathrm{Hg}$ inorganique, la distribution est déjà à l'équilibre après seulement dix jours d'expérience. Ceci montre que le mercure inorganique voyage vite, occupe un nombre déterminé de sites et s'équilibre entre les organes à un niveau relatif qui devient rapidement indépendant des quantités reçues quotidiennement. Ce dernier point est confirmé par l'absence de corrélation entre le pourcentage de rétention et le temps écoulé. Les données de charges relatives pour le $\mathrm{MeHg}$ sont très révélatrices du comportement particulier de cet organométal. Ces données indiquent un entreposage progressif du MeHg dans l'endosquelette et dans les gonades. Contrairement au $\mathrm{Hg}$ inorganique, le MeHg voyage lentement, occupe un nombre toujours croissant de sites dans les gonades et l'endosquelette et ne s'équilibre pas dans les différents compartiments de l'organisme. Le taux de rétention qui augmente continuellement au cours de l'expérience est aussi révélateur de la capacité du $\mathrm{MeHg}$ d'occuper un nombre considérable de sites. Ceci indiquerait aussi une moins grande spécificité des liaisons du $\mathrm{MeHg}$ par rapport au $\mathrm{Hg}$ inorganique. En d'autres mots, le MeHg exerce une grande affinité pour un grand nombre de sites divers qu'il occupe pour peu qu'il puisse y parvenir. L'allure de sa courbe de bioaccumulation (fig. 1) semble aussi suggérer la présence d'un deuxième palier au-dessus de celui déjà discuté par le modèle cinétique. Dans ce cas précis, il est possible qu'une deuxième série de sites, plus difficilement accessibles à cause 
d'une compétition plus forte et d'une plus faible affinité, deviennent disponibles avec l'augmentation constante de la charge globale. En cas de cessation de la contamination, ces sites seraient rapidement nettoyés, les compétiteurs ioniques (si on s'en tient au présent modèle d'échanges ioniques) reprenant rapidement leurs places. Le MeHg s'échange donc plus rapidement entre la solution et les sites (et entre les sites eux-mêmes) et semble ainsi mettre un peu plus de temps à se bioaccumuler du moins pour le premier niveau d'adsorption. Dans une étude sur l'adsorption et la désorption du mercure sur des érythrocytes, RABEISTEIN et al. (1983) avaient aussi remarqué la plus grande vitesse d'échange du méthylmercure à la surface de cellules biologiques.

En conclusion, ces données nous montrent qu'il existe une relation chimique très étroite entre la source et le tissu récepteur dans le processus de prise en charge des espèces de mercure. L'étoile de mer est particulièrement pratique pour cette démonstration puisqu'il n'y a pas de production de déchets solides à prendre en compte dans le modèle cinétique. Un mécanisme d'adsorption par échange ionique semble bien représenter le processus de bioaccumulation de deux espèces de mercure dans les caeca pyloriques de l'étoile et une constante de vitesse légèrement supérieure pour le $\mathrm{MeHg}$ a pu être calculée quoique le mécanisme ne soit pas entièrement de nature ionique pour le $\mathrm{MeHg}$. L'étoile de mer, par sa grande efficacité à digérer tout ce qui est ingéré, semble en mesure de jouer un rôle important à la fois dans la séquestration du méthylmercure (presque $100 \%$ de rétention après 40 jours) et dans la remise en solution du mercure inorganique (autour de $50 \%$ du total ingéré). A notre connaissance, aucune donnée similaire n'existe pour d'autres métaux traces mais le rôle potentiel de l'étoile de mer et des autres échinodermes dans le cycle biogéochimique du mercure en milieu côtier doit être exploré. L'étoile de mer est une sorte de "digesteur marin " qui engouffre des bivaives et d'autres invertébrés et diffuse des métabolites solubles et des métaux traces qui n'ont pas été bioaccumulés. A cause de leur biomasse souvent élevée, de leur abondance le long de certaines côtes et de leur longévité, il devient important d'examiner de près leur contribution et celle des autres échinodermes au cycle des métaux traces dans les eaux côtières.

\section{RÉFÉRENCES BIBLIOGRAPHIQUES}

BJERREGARD P., 1988. Effect of selenium on cadmium uptake in selected benthic invertebrates. Mar. Ecol. Prog. Ser., 48, 17-28.

DEN BESTEN P.J., HERWIG H.J., ZANDEE D.I. VOOGT P.A., 1989. Cadmium accumulation and metallotionein-like proteins in the sea star Asterias rubens L. Mar. Environ. Res., 28, 163-166.

GUARY J.C., FAWDER S.W., BEASLEY M., 1982. Routes of plutonium uptake and their relation to biomagnification in starfish. Mar. Pollut. Bull., 13, 99-102.
HATCH W.R., L.W. OTT, 1968. Determination of submicrogram quantities of mercury by atomic adsorption spectroscopy. Anal. Chem., 40, 2085-2087.

IGLESIAS N., PENCHASZADECH P.E., 1983. Mercury in the sea star from Golfo Triste, Venezuela. Mar. Pollut. Bull, 14, 396-398.

MAHEU S., PELLETIER E., 1995. Effects of complexing agents on the distribution of $\mathrm{Hg}$ (11) species provided by food to the starfish Leptasterias polaris. Chem. Spec. Bioavail., 6, 103-112. 
MISRA D.N., BOWEN R.L., 1981. Interaction of zinc ions with hydroxylapatite. In "Adsorption from aqueous solutions". TEWARI P.H. ed. Plenum Press, New York, 179-192.

PALMER S.J., PRESLEY B.J., TAYLOR R. J., POWELL E.N., 1993. Field studies using the oyster Crassostrea virginica to determine mercury accumulation and depuration rates. Bull. Environ. Contam. Toxicol., $51,464-470$.

PELLETIER E., 1986. Modification de la bioaccumulation du sélénium chez Mytilus edulis en présence de mercure organique et inorganique. Can. J. Fish. Aquat. Sci., 43, 203-210.

PELLETIER E., 1995. Environmental organometallic chemistry of mercury, tin, and lead : Present status and perspectives. In * Metal Speciation and Bioavalablity in Aquatic Systems ", TESSIER, A. et D.R. TURNER, Ed., IUPAC Series, John Wiley \& Sons, London, 103-148

PELLETIER E., LAROCQUE R., 1987. Bioaccumulation of methymercury in starfish from contaminated mussels. Mar. Pollut. Bull., $18,482-485$.

RABEINSTEIN D.L., REID R. S, ISAAB A.A., 1983. ${ }^{1} H$ NMR study of the effectiveness of various thiols for removal of methylmercury from hemolysed erythrocytes. J. inorg. Biochem., 18, 241-251.

RIISGARD H.U., KIORBOE T., MOHLENBERG F., DRABAEK I., PHEIFFER MADSEN P., 1985. Accumulation, elimination and chemical speciation of mercury in the bivalves Mytilus edulis and Macoma balthica. Mar. Biol., 86, 55-62.

RIISGARD H.U., HANSEN S. 1990. Biomagnification of mercury in marine grazing foodchain : algal cells Phaeodacylum tricornutum, mussels Mytilus edulis and flounders Platichthys flesus studied by means of a
stepwise-reduction-CVAA method. Mar. Ecol. Prog. Ser., 62, 259-270.

RIISGARO H.U., FAMME P., 1986. Accumulation of inorganic and organic mercury in shrimp. Mar. Pollut. Bull., 17, 255-257

ROULEAU C., PELLETIER E., PELLERINMASSICOTTE J., 1992. Uptake of inorganic mercury and selenium from food by the nordic shrimp Pandalus borealis Chem. Spec. Bioavail., 4, 75-81.

ROULEAU C., PELLETIER E., TJaLVE H. 1995. Distribution kinetics of single-doses of methylmercury, tributyltin and corresponding inorganic ions in starfish Leptas terias polaris. Mar. Ecol. Prog. Ser., 124, 143-158.

SORENSEN M., BJERREGARD P., 1991. Interaction between accumulation of mercury and selenium in the starfish Asterias rubens. Mar. Biol., 108, 269-276.

TESSIER A., BUFFLE J., CAMPBELL P.G.C., 1994. Uptake of trace metals by aquatic organisms. In "Chemical and Biological Regulation of Aquatic Systems", BUF. FLE J., DEVITRE R., Ed, Lewis Publishers, London, 199-232.

WARNAU M., LEDENT G., TEMARA A., ALVA V., JANGOUX M., DUBOIS Ph., 1995a. Allometry of heavy metal bioconcentration in the Echinoid Paracentrotus lividus. Arch. Environ. Contam. Toxicol. 29, 393-399.

WARNAU M., LEDENT G., TEMARA A., JANGOUX M., DUBOIS Ph., 1995b. Experimental cadmium contamination of the echinoid Paracentrotus lividus: influence of exposure mode abnd distribution of the metal in the organism. Mar. Ecol. Prog. Ser. 116, 117-124.

WRIGHT D.A., WALBOURNE P.M., MARTIN A.M.V., 1991. Inorganic and organic mercury uptake and loss from the crayfish Oronectes propinquus. Water Air Soil Pollut., 56, 697-708. 